\title{
Stability study for matching in laser driven plasma acceleration
}

\author{
A.R. Rossi ${ }^{\text {a,*, M.P. Anania }}{ }^{\mathrm{b}}$, A. Bacci ${ }^{\mathrm{a}}$, M. Belleveglia ${ }^{\mathrm{b}}$, F.G. Bisesto ${ }^{\mathrm{b}}$, E. Chiadroni $^{\mathrm{b}}$,

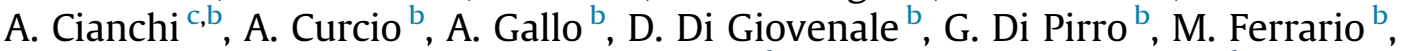

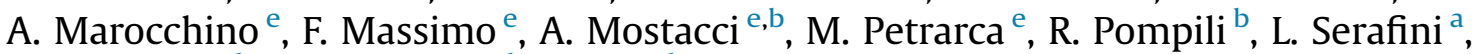 \\ P. Tomassini ${ }^{\mathrm{d}}$, C. Vaccarezza ${ }^{\mathrm{b}}$, F. Villa ${ }^{\mathrm{b}}$ \\ a INFN - MI, via Celoria 16, 20133 Milan, Italy \\ ${ }^{\mathrm{b}}$ INFN - LNF, v.le E. Fermi, 00044 Frascati, Italy \\ ' Tor Vergata University, Physics Department, via della Ricerca Scientifica 1, 00133 Rome, Italy \\ d University of Milan, Physics Department, via Celoria 16, 20133 Milan, Italy \\ e La Sapienza University, SBAI Department, via A. Scarpa 14, 00161 Rome, Italy
}

\section{A R T I C L E I N F O}

Available online 12 February 2016

Keywords:

Laser Wakefield Acceleration

Electron beam dynamics

Electron beam matching

\begin{abstract}
A B S T R A C T
In a recent paper [14], a scheme for inserting and extracting high brightness electron beams to/from a plasma based acceleration stage was presented and proved to be effective with an ideal bi-Gaussian beam, as could be delivered by a conventional photo-injector. In this paper, we extend that study, assessing the method stability against some jitters in the properties of the injected beam. We find that the effects of jitters in Twiss parameters are not symmetric in results; we find a promising configuration that yields better performances than the setting proposed in [14]. Moreover we show and interpret what happens when the beam charge profiles are modified.
\end{abstract}

(c) 2016 Elsevier B.V. All rights reserved.

\section{Introduction}

The basic principles of Laser WakeField Acceleration (LWFA) [1] have been extensively studied and are routinely exploited worldwide [2]; plasma based acceleration is potentially one of the most promising techniques to build next generation, compact and cheap, high energy accelerators. However there still exist a considerable lag between the exploitation of plasma based acceleration and the practical realization of a working plasma accelerator.

The main difficulties, from a beam dynamics point of view, arise because of the extremely intense transverse fields present in plasmas. Among the problems generated by this simple feature we can list: a high sensitivity to jitters and asymmetries [3]; very demanding requirements for matching beams in plasma channels [4]; a large normalized emittance degradation occurring whenever the beam propagates through a conventional beam line downstream the plasma acceleration stage [5], even if this means a trivial drift in vacuum. This last phenomenon is rooted in the intrinsic properties of an electron bunch accelerated by plasma: because of the small scales lengths of plasma waves and of the intense focusing fields, the accelerated beam usually possess a

\footnotetext{
* Corresponding author.

E-mail address: andrea.rossi@mi.infn.it (A.R. Rossi).
}

rather large energy spread and high divergence [6,7]. This makes their management extremely awkward.

The solutions proposed so far make use of plasmas as optical elements (lenses) in order either to propagate the bunches from plasma acceleration to a "user" experiment or to couple different acceleration stages. It is possible to divide the conceived plasma optical devices in active and passive lenses: the former need some kind of independent power supply, whereas the latter are powered by the same driver that produces the accelerating plasma wave. Lenses belonging to the first category, such as the one reported in [8] and the "double-pulse" lens of [9], are usually conceived as stand alone devices: this implies some amount of drift, between the plasma acceleration stage and the lenses, that could potentially spoil beam properties before reaching the plasma optics; moreover synchronization is required, although this may not constitute a great difficulty. As for passive lenses, they can be both stand alone elements, such as the "single-pulse" lens of [9], or integrated devices. This means that they can be implemented as extensions of already needed devices, such as capillaries, gas-jets or plasma cells. The operating principles of passive-integrated plasma lenses have been suggested some years ago $[5,7,10,11]$ and are based on a longitudinal tapering of plasma density; further investigations showed they can be successfully operated both in the bubble regime of the plasma wave [12] and, together with a judicious choice of laser focusing, in the linear regime [13], provided beam loading is negligible. In [13] it was also shown how the longitudinal tapering can reduce detrimental effects due to offsets in beam alignment. 
In a recent paper [14], some of us demonstrated that the method of combining longitudinal tapering and laser focusing can yield interesting results also in a more appealing setting, where beam loading is not negligible and the plasma wave is in the quasilinear regime. Use of passive, integrated solutions seems to constitute, at least in simulations, a choice that can match the performances of active lenses, with the further advantage of an easier implementation and operation.

In this paper we extend the numerical study presented in [14], in order to verify the stability of the results with respect to the beam charge density profiles and errors in the initial matching conditions in plasma. Moreover a couple of interesting features of beam dynamics are identified and analyzed, extending and integrating what was reported in [14].

The paper is organized as follows: in Section 2 we briefly review the results of [14], defining the reference setting, and showing how the beam transport downstream the plasma is less troublesome when the beam is properly extracted from the plasma; in Section 3 the results of the stability study are presented and commented. Moreover, we give some estimations on the jitters in matching conditions that can be tolerated; in Section 4 we present an heuristic explanation of interesting features shown by emittance evolution in some instances; finally, in Section 5 we draw some conclusions.

\section{Matching of electron bunches to/from a plasma stage}

The matching condition for a given beam in any focusing system, can be derived starting from the transverse envelope equation. Assuming cylindrical symmetry, the matched size in a plasma channel turns out to be [4]:

$\sigma_{\text {tr,match }}=\sqrt[4]{\frac{2}{\gamma}} \sqrt{\frac{\varepsilon_{n}}{k_{p}}}$

where $\varepsilon_{n}$ is the normalized phase space emittance, $k_{p}^{2}=4 \pi n_{0} e^{2} /$ $m c^{2}$ the plasma wavenumber, $n_{0}$ the unperturbed plasma density, $\gamma$ the beam average Lorentz factor, $e$ is the electron charge, $m$ its rest mass and $c$ the speed of light. All analytical results derived so far $[13,15,16]$ are exact only either when the focusing strength is constant along the bunch (i.e. in the bubble regime) or in the linear regime; moreover beam loading is considered negligible. The derivation of 1 implies that the beam Twiss parameter $\alpha$ is zero [17].

When Eq. (2) is evaluated for a typical high brightness beam delivered by a photoinjector $\left(E \approx 100 \mathrm{MeV}, \varepsilon_{n} \approx 1 \mu \mathrm{m}\right)$ the matched size turns out to be $\sigma_{\text {tr,match }} \approx 1.3 \mu \mathrm{m}$ for a plasma density $n_{0}=10^{17} \mathrm{~cm}^{-3}$; this density value is considered to be a good choice in planned and ongoing external injection experiments [18,19]. Such a small spot-size for a high brightness beam requires a matched Twiss beta function of the order of few hundreds of $\mu \mathrm{m}$, an unfeasible task even for state of the art permanent magnets quadrupoles [20].

The exploitation of plasma tapering (plasma ramps) and laser focusing allows us to gently bring the beam transverse size $\sigma_{\text {tr }}$ from a value manageable by conventional beam lines optics, to the tight focusing needed in plasma acceleration stages; the reverse is true when the beam leaves the plasma and needs to be coupled to an experimental line or another stage of plasma acceleration. If an adiabatic matching [16] is realized, those processes occur without a significant emittance increase, as shown in [13] with numerical simulations. This requires the plasma ramps to be much longer than the electrons betatron wavelength $\lambda_{\beta}=\sqrt{2 \gamma} \lambda_{p}$, where $\lambda_{p}=2 \pi / k_{p}$. The effective ramp length can be shortened if a specific, optimized tapering profile could be implemented [16]; however, different profiles can still provide adiabatic matching if the ramp length is increased accordingly.
Table 1

Input bunch parameters. The reported transverse size is the one attaining matching.

\begin{tabular}{ll}
\hline Charge & $10 \mathrm{pC}$ \\
\hline$\sigma_{x, y}$ & $1.3 \mu \mathrm{m}$ \\
$\sigma_{z}$ & $2 \mu \mathrm{m}$ \\
$\epsilon_{x, y}$ & $1 \mu \mathrm{m}$ \\
Energy & $80 \mathrm{MeV}$ \\
$\Delta E / E$ & $0.2 \%$ \\
\hline
\end{tabular}

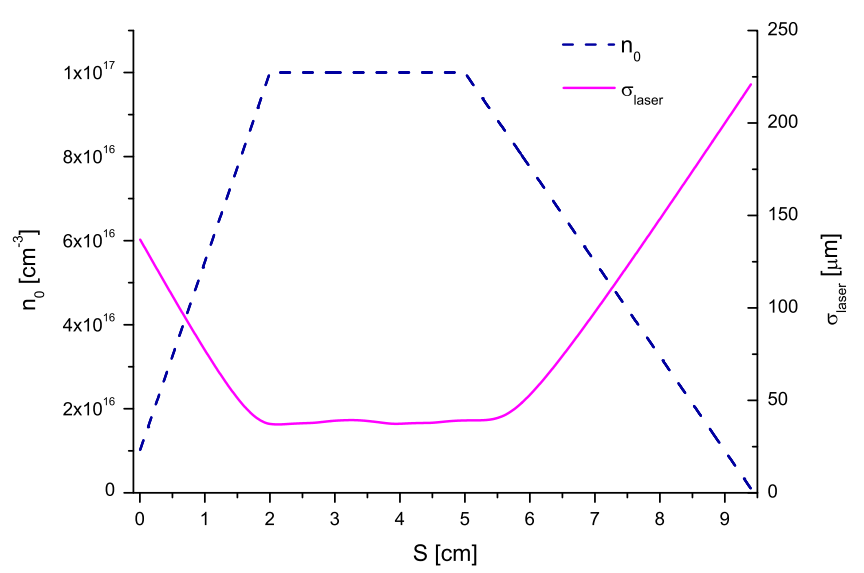

Fig. 1. Laser average radius (solid line) and plasma density profile (dashed line). The three stages of the transport (insertion, acceleration, extraction) with relative lengths and densities. Throughout acceleration the laser is guided by the capillary, while it evolves freely in both matching sections. (For interpretation of the references to color in this figure caption, the reader is referred to the web version of this paper.)

In [14] we showed how it is possible to perform the matching task, for a bi-Gaussian beam whose properties are summarized in Table 1, employing an experimental setting as shown in Fig. 1 (blue line). Such a plasma density configuration could be produced by proper shaping of the capillary geometry and tuning the pressure of the gas applied to the inlet(s). An insertion linear plasma ramp of length $L_{1}=2.0 \mathrm{~cm}$, and plasma density varying from $n_{\mathrm{i}}=$ $10^{16} \mathrm{~cm}^{-3}$ to $n_{0}=10^{17} \mathrm{~cm}^{-3}$, brings the beam to an acceleration stage whose length $L_{\text {acc }}$ is $3 \mathrm{~cm}$; after acceleration, the bunch is coupled back to vacuum by means of an extraction ramp with length $L_{2}=4.4 \mathrm{~cm}$ and density going from $n_{0}$ to $n_{f}=10^{15} \mathrm{~cm}^{-3}$. In the accelerating stage, the plasma wavelength is $\lambda_{p} \approx 100 \mu \mathrm{m}$. We used linear ramps for sake of simplicity. The distance between the barycenters of driver and witness is set to be $\Delta z=50 \mu \mathrm{m}$.

A laser pulse with $\lambda_{\mathrm{L}}=800 \mathrm{~nm}$ drives the plasma wave. The pulse is Gaussian transversally, while the longitudinal profile is a squared sine. It is focused at the beginning of the acceleration stage, Fig. 1 magenta line, down to an rms size $\sigma_{\mathrm{L}}=38.6 \mu \mathrm{m}$ in order to be matched in a dielectric capillary hollow waveguide whose inner radius is $R_{\text {cap }}=60 \mu \mathrm{m}$ [21]. After guiding in the capillary, the laser is allowed to defocus freely in the extraction ramp. The pulse length is $35 \mathrm{fs}$ FWHM and the energy is $3.5 \mathrm{~J}$, so that at focus we have $a_{0} \approx 1.4$. This value allows us to drive a quasi-linear plasma wave. The choice of laser and capillary parameters has been detailed in [18].

For simulations, we used the hybrid fluid/Particle in Cell (PIC) code QFLUID [18]. The plasma component is modeled as a cold fluid while the accelerated bunch is fully kinetic. Cylindrical symmetry is assumed and the laser evolves under the envelope approximation. QFLUID makes also use of the quasi-static approximation and evolves the plasma component by solving 
(a)

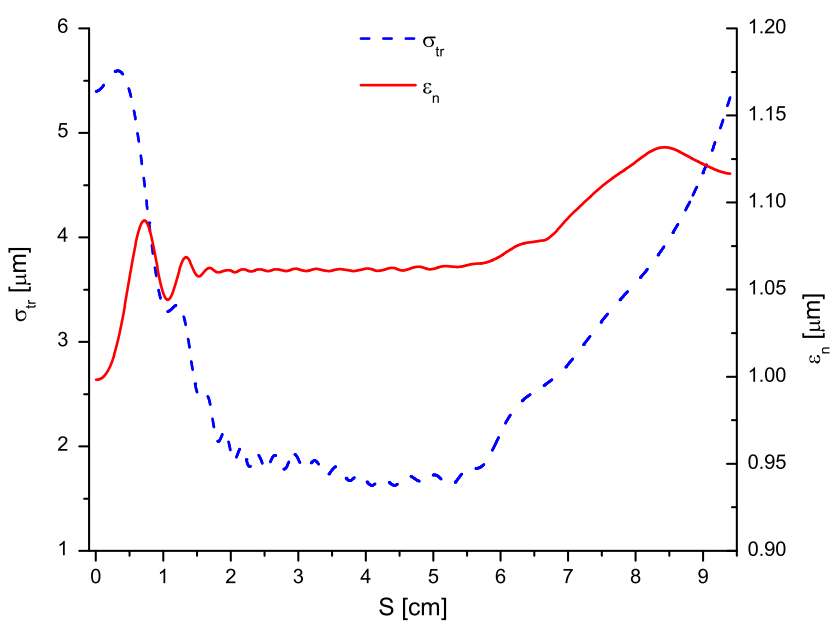

(b)

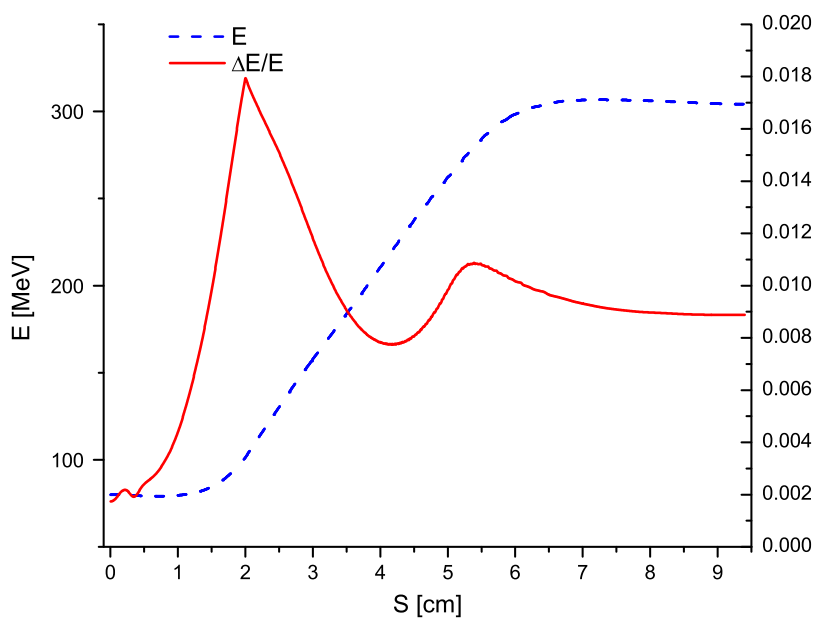

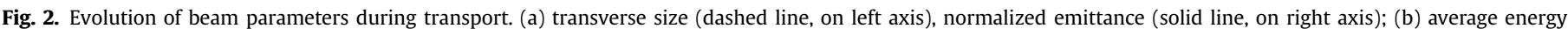

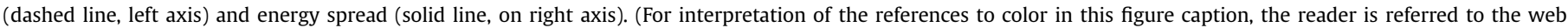
version of this paper.)

(a)

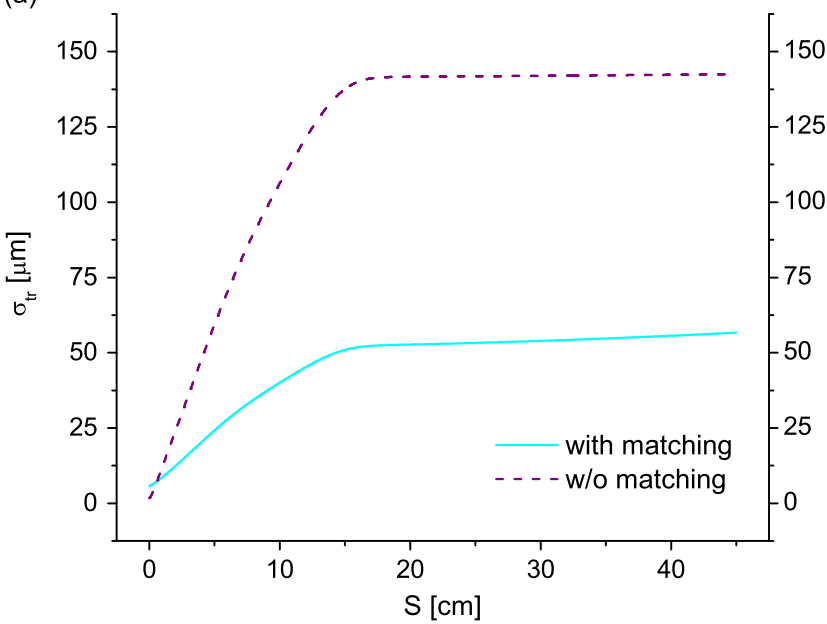

(b)

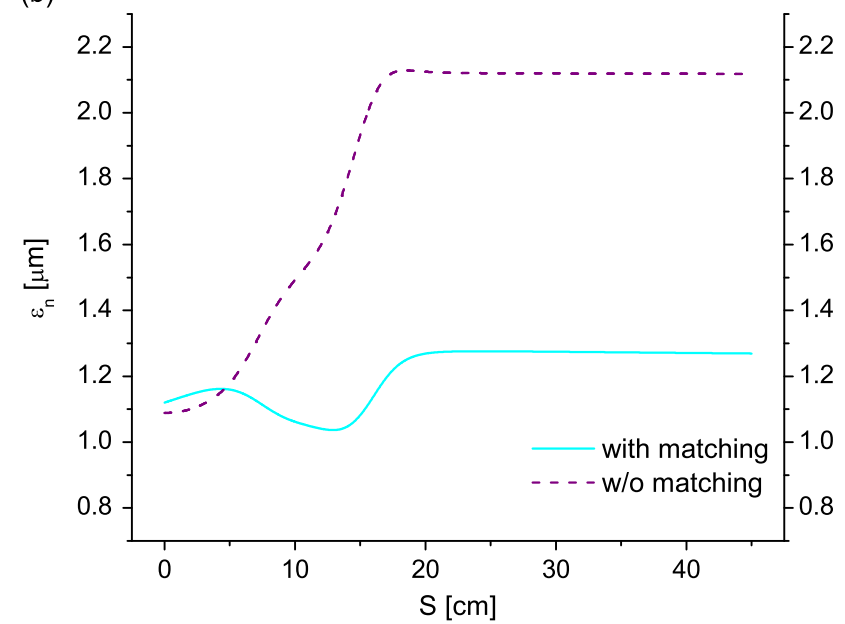

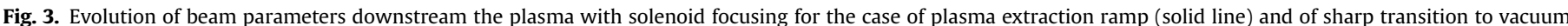

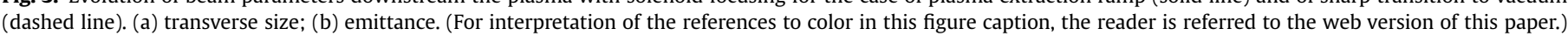

the partial differential equation reported in the appendix of [22]. It has been benchmarked against the PIC code ALaDyn [23].

The transverse beam parameters evolution is reported in Fig. 2(a). A nice matching is shown by the transverse size $\sigma_{\mathrm{tr}}$ evolution (blue line) where small ripples are due to the rms nature of matching conditions for a Gaussian current profile; nevertheless $\sigma_{\mathrm{tr}}$ is brought from an injection value of around $5.5 \mu \mathrm{m}$ down to the matched value in the acceleration stage, $\sigma_{\mathrm{tr}}$ match $\approx 2 \mu \mathrm{m}$, and back to the initial value, without a significant emittance dilution (red line), since its final value is increased by only $\approx 10 \%$. The evolution of energy and energy spread are shown in Fig. 2(b) (blue and red lines, respectively). The total energy increase is around $220 \mathrm{MeV}$, implying an effective accelerating gradient in excess of $7 \mathrm{GV} / \mathrm{m}$ within the $3 \mathrm{~cm}$ acceleration stage. Most importantly, the final energy spread is kept under the $1 \%$ threshold, which guarantees a contained emittance dilution due to chromatic effects in subsequent drifts.

The results shown so far still need a deeper optimization, since the final value of the $\alpha_{\mathrm{T}}$ Twiss parameter is around -0.7 , whereas it is argued in [16] that a better value should be $\alpha \lesssim-1$. Nevertheless the effects due to the extraction ramp can be readily quantified. To this extent, we simulated the transport downstream the plasma using Astra [24]. In a first run, the starting condition was that of the bunch at the end of the extraction $\operatorname{ramp}(s=9.4 \mathrm{~cm})$; then we repeated the simulation but using the bunch at a distance of $s=5.0 \mathrm{~cm}$ from the beginning of the insertion ramp, i.e. as if there was a sharp transition between the acceleration stage and vacuum. With the goal of producing a constant envelope in the subsequent drift, we placed a $18 \mathrm{~cm}$ long solenoidal field at $5.0 \mathrm{~cm}$ from the starting position. The choice of a solenoid was dictated only by simplicity and symmetry considerations: the peak field value $B_{0} \approx$ $20 \mathrm{~T}$ needed for flattening the envelope evolution is unrealistically high; however, the resulting focusing strength is easily achievable with a doublet of permanent magnets quadrupoles.

Simulation results are reported in Fig. 3, both for the ramp extracted beam (cyan lines) and for the unmatched beam (purple lines). Fig. 3(a) shows that in both situations we managed to have a flat envelope evolution; notice how the two asymptotic values differ in magnitude by a factor $\approx 2$. The same is true for the emittance evolution, where the ramp extracted beam experiences a dilution smaller than $20 \%$, resulting in a final value smaller than $1.3 \mu \mathrm{m}$, whereas the unmatched one nearly doubles its emittance. It is worth to notice that the differences in the transports cannot be ascribed to chromatic effects, since the energy spread starting 
(a)

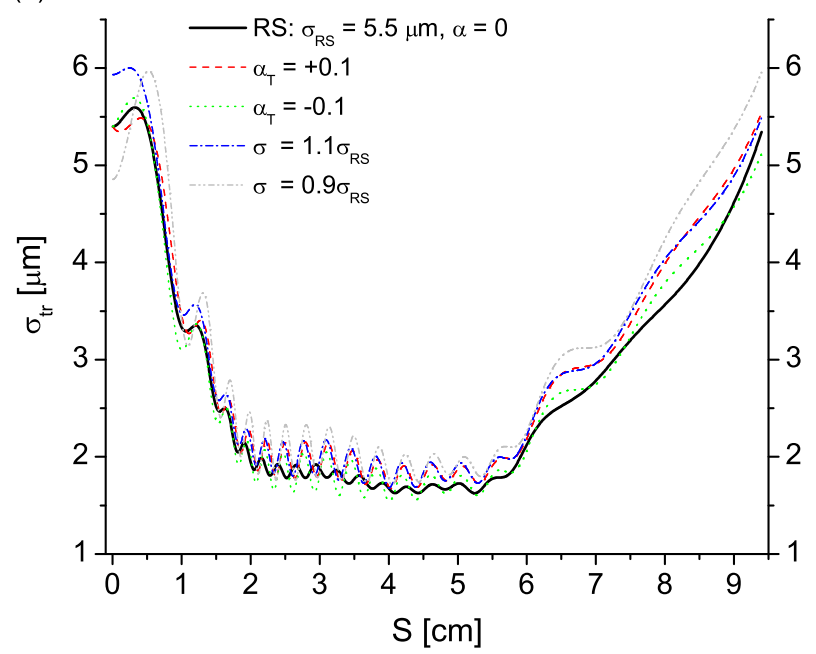

(b)

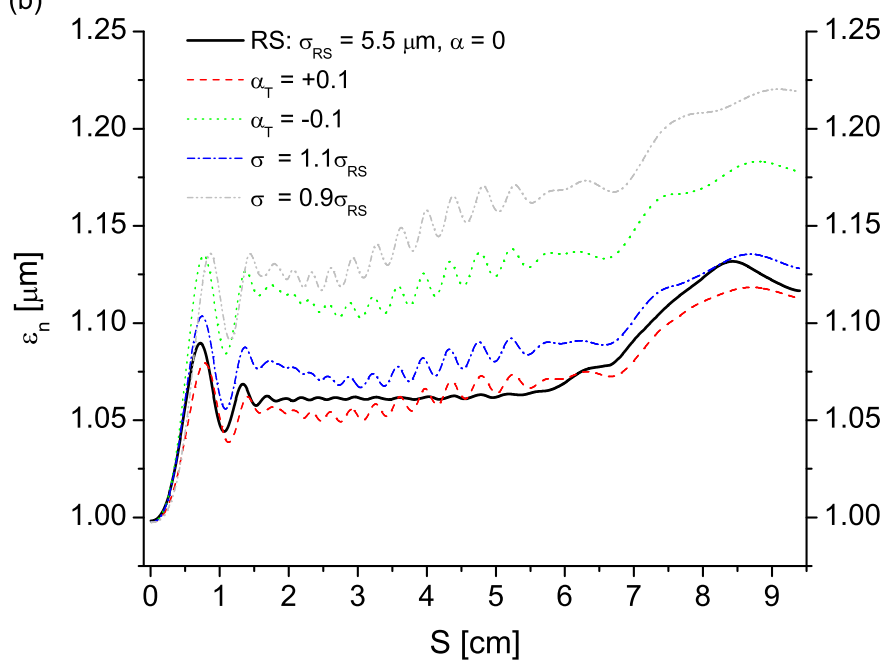

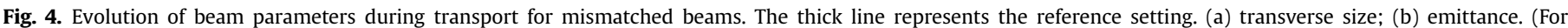
interpretation of the references to color in this figure caption, the reader is referred to the web version of this paper.)

values at $s=5.0 \mathrm{~cm}$ and $s=9.4 \mathrm{~cm}$ are very close, as can be deduced from Fig. 2(b). These results demonstrate in a conclusive way that the quasi-adiabatic extraction is as important as matching at insertion.

\section{Stability of the matching strategy}

The results shown in Section 2 represent our reference setting (RS). Since the matching conditions established are still quite demanding, we would like to asses the stability of the solution found with respect to small deviations from the RS injection parameters in the insertion ramp. To this end, we ran simulations using QFLUID slightly modifying either the value of $\sigma_{\text {tr }}$ by $\pm 10 \%$ or of $\alpha_{\mathrm{T}}$ by \pm 0.1 . These values can be attributed to an uncertainty in final focusing strength of about $10 \%$ or a misplacement of the beam waist of $\pm 0.5 \mathrm{~mm}$. Results for the evolution of transverse size and emittance are reported in Fig. 4.

The evolution of transverse size, Fig. 4(a), does not display large differences in any run, except for the amplitude of ripples along the transport. The final beam size is spread in an interval of around $0.5 \mu \mathrm{m}$ which constitutes roughly $10 \%$ of the initial matched value.

Emittance evolution, Fig. 4(b), reveals that the performances are not symmetrical around the matched conditions: a bunch whose focusing is too tight (gray line) or is diverging while entering the insertion ramp (green line) undergoes a larger emittance dilution with respect to a slightly larger beam (blue line) or a converging beam (red line). This last situation, indeed, seems to yield a comparable result, if not better, as the RS.

Regarding longitudinal parameters of the bunch, they turn out to be very stable, as expected, since the injection phase in the plasma wave was not changed. The differences in final energy and energy spread of the situations reported in Fig. 4 can all be attributed to numerical approximations.

Another difference that can arise in an experimental setting resides in the exact profiles of the charge distribution within the bunch and in the details of the longitudinal phase space. The chosen bi-Gaussian (BG) profile is correct, strictly speaking, only when space charge forces can be neglected with respect to emittance pressure. In a relatively low energy $(\lesssim 100 \mathrm{MeV})$ high brightness beam, with peak current in the range of $1 \mathrm{kA}$, our assumption could be too naive. Moreover, if the injected bunch comes from a magnetic compression stage, it may possess a linear energy chirp. In order to investigate how these differences can modify the plasma stage performances, we modified our incoming beam as follows. First, we simulated a beer can (BC) charge profile, i.e. a flat top distribution both in longitudinal and transverse directions, with all rms parameters equal to RS (Fig. 5, red line); second, the uncorrelated energy spread of the BC distribution was raised to be $1 \%$ (Fig. 5, green line); third we modified RS to have a correlated energy spread of $1.3 \%$ (uncorrelated energy spread unchanged) with a chirp of $\pm 0.25 \mathrm{MeV} / \mu \mathrm{m}$ (Fig. 5, cyan and magenta lines). The $\mathrm{BC}$ distribution represents the theoretical configuration of a beam when space charge dominates emittance pressure (i.e. the opposite limit with respect to BG distribution) while the chirp value represents a quite typical outcome when the bunch is magnetically compressed. As before, final envelope values do not differ more than $10 \%$ from the RS final result, so they are not reported; energy spread always results lower than $1 \%$ because of the longitudinal phase space rotation performed by plasma fields and cooling due to acceleration [17]. From Fig. 5 it appears that the $\mathrm{BC}$ distribution yields better results in term of emittance compared to the BG distribution. Upon inspection, the lower emittance values can be ascribed to a better emittance compensation in the extraction ramp [14]; this fact is due to the different way the $\mathrm{BC}$ distribution loads the wake, in particular at the very beginning and the very end of transport, when beam loading dominates; we will elaborate more on that in Section 4. The almost coincident results on the emittance evolution of the high and low uncorrelated energy spread $\mathrm{BC}$ distributions seems to indicate that the analyzed matching and acceleration scheme is not very sensitive to uncorrelated $\Delta E / E$; this is not surprising because of the high intensity of the longitudinal plasma field, that completely overcomes stochastic features.

The same is true for both BG distributions with a chirp that perform as the RS even reversing the sign of the chirp. This proves the insensitivity of final results to small variations in the exact features of the initial longitudinal phase space, at least for the beam parameters under examination.

Our results are quite setting dependent but we expect them to hold true at least in a neighborhood of initial energy and emittance values (and, possibly, also charge), since we showed that the starting conditions of the RS do not reside in an unstable equilibrium point. What can be safely stated is that, increasing the initial and/or final energy would require an increase of the insertion/ extraction ramp length, due to the bond between betatron wavelength and energy; moreover, both a variation in energy and of 
initial emittance requires to modify the initial beam size accordingly, following the indications of (1).

\section{Beam dynamics in matching and acceleration sections}

In the preceding sections, we noticed how a Twiss $\alpha_{\mathrm{T}}$ parameter slightly greater than zero could improve transport and stated that the better performances of the $\mathrm{BC}$ distributions could be attributed to the different way they load the wake. Both results, together with the emittance compensation effect in the final extraction ramp, can be explained by the interplay of beam loading and electrons dynamics.

We already explained heuristically in [14] how the emittance compensation can be attributed to particles belonging to head and lateral periphery of the bunch. When beam loading effects dominate over driver induced plasma fields in the ramp, those particles start to drift in a region where the plasma is almost neutral and the transverse force weak, as shown in Fig. 6(a) that reports the

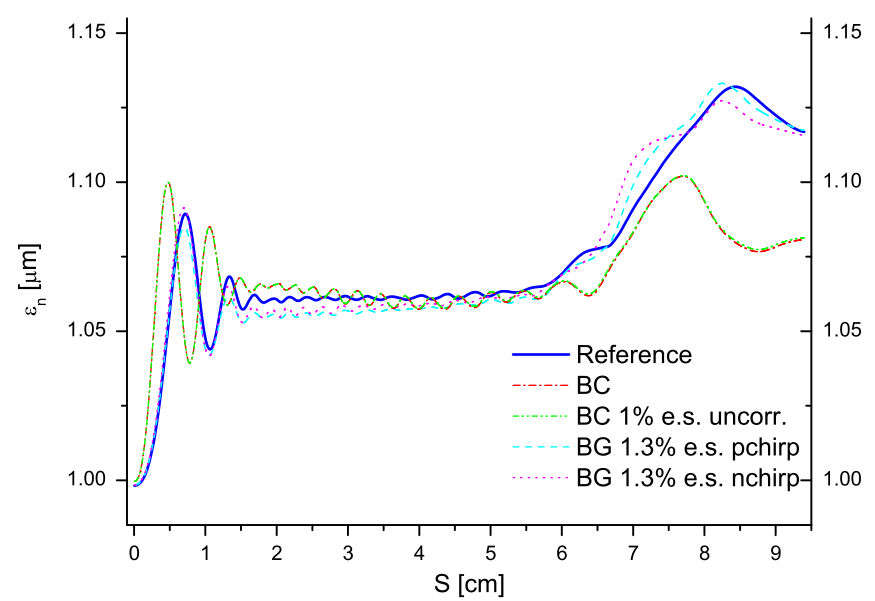

Fig. 5. Emittance evolution for different bunch charge distributions and longitudinal phase spaces. See main text for details. (For interpretation of the references to color in this figure caption, the reader is referred to the web version of this paper.)

(a)

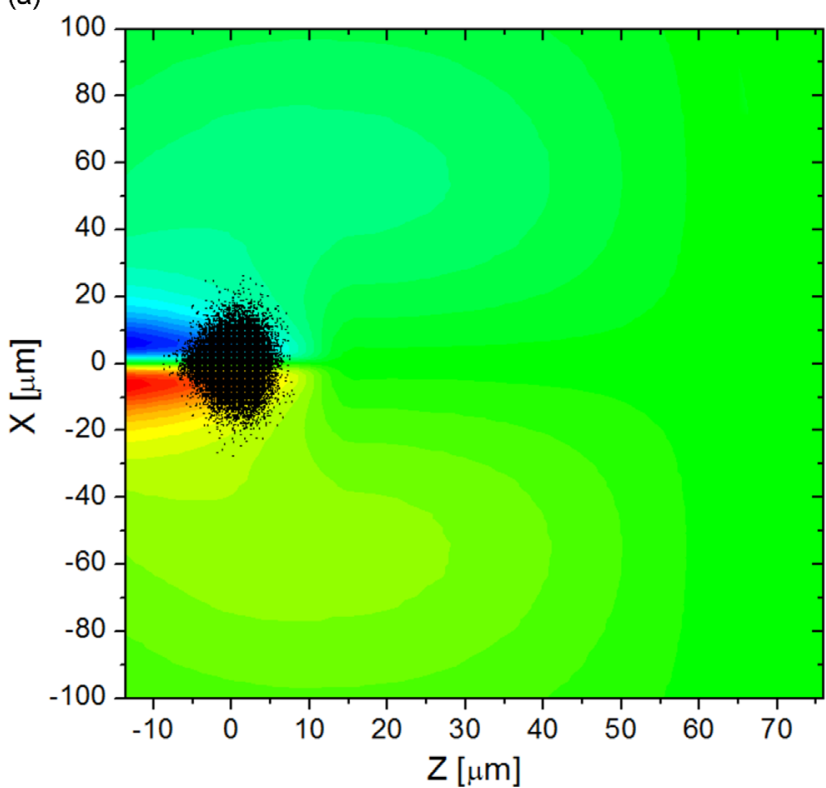

value (in arbitrary units) of the transverse force at a distance of about $4 \mathrm{~mm}$ from injection. There is evident how the laser generated fields are much weaker than the beam loading effects; moreover, the beam induced fields have the transverse extent of the beam r.m.s. dimension and become very intense within the beam itself, at a position about $\sigma_{z}$ in front of the peak current. Beyond those boundaries, in the transition region from beam induced an laser induced fields, the focusing force has a non-linear behavior, resulting in halo formation. On the contrary, laser induced fields have a much larger radial extent $(\approx 100 \mu \mathrm{m}$ in radius) and are always linear in the bunch region. Within the insertion ramp, the particles that drifted radially away from the axis are eventually brought back in the accelerating/focusing region of the plasma wave, since the wake generated by the laser always remains much larger, in the subsequent transport, than the dimension of the bunch itself: this can be seen in Fig. 6(b), where the transverse force is plotted at a distance $s \approx 33.5 \mathrm{~mm}$. It appears that the whole bunch is kept focused by the laser induced transverse force that completely overcomes any beam loading effect. This partially explains the presence of an emittance peak within the first $10 \mathrm{~mm}$ of the insertion ramp and why emittance value is reduced thereafter. We would like to stress that the effect is different from the emittance compensation exploited in high brightness injectors [25]. There, different longitudinal slices rotate with different betatron frequency within a solenoid and eventually get re-aligned in the subsequent waist, producing an emittance compensation. Here, the 'slices' should not be understood to be mainly in the longitudinal direction but in the transverse. To show the dynamics is different, we sliced our bunch in five longitudinal parts of equal length, calculated for each slice the value of $\alpha_{\mathrm{T}}$ and reported its variance $\Delta \alpha_{\mathrm{T}}$, using the charge content of each slice as statistical weight. The result is reported in Fig. 7. In the emittance 'longitudinal' compensation process one expects to find a one-toone correspondence between $\Delta \alpha_{\mathrm{T}}$ and emittance, whereas in Fig. 7 it is not the case: all curves peak around $1 \mathrm{~cm}$ while the emittance peak for all BC distributions is before that value. Moreover, the $\Delta \alpha_{\mathrm{T}}$ variation in the last 2 centimeters is inconsistent with the emittance reduction of Fig. 5. The large difference between $B C$ and $B G$ distributions is due to the different charge content of corresponding slices (i.e. the presence of low charge

(b)

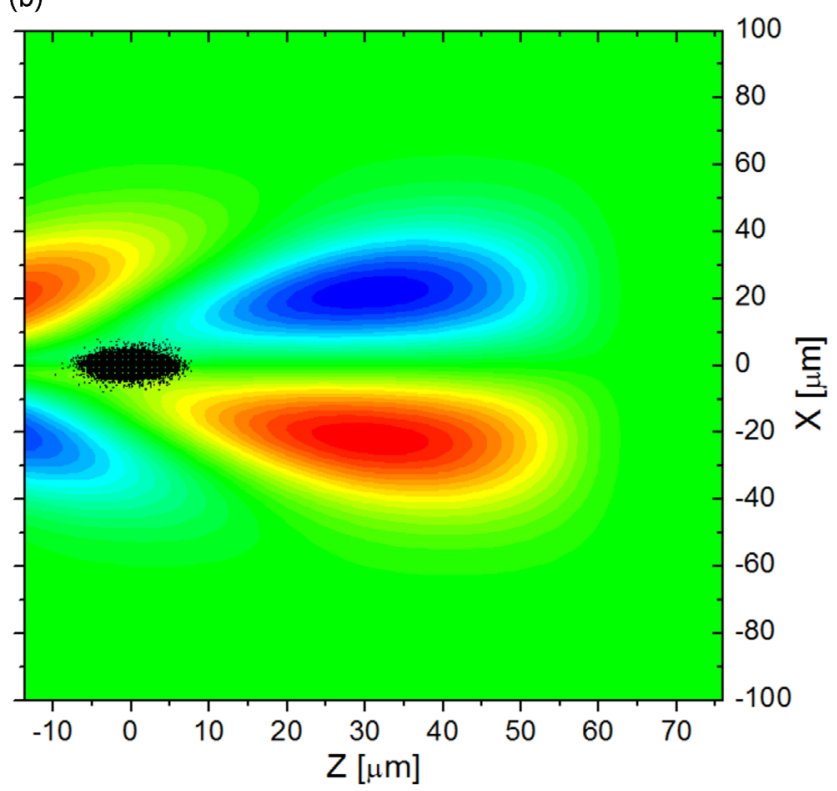

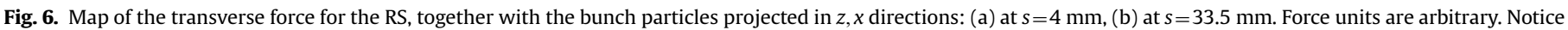
that plots have different scales. 


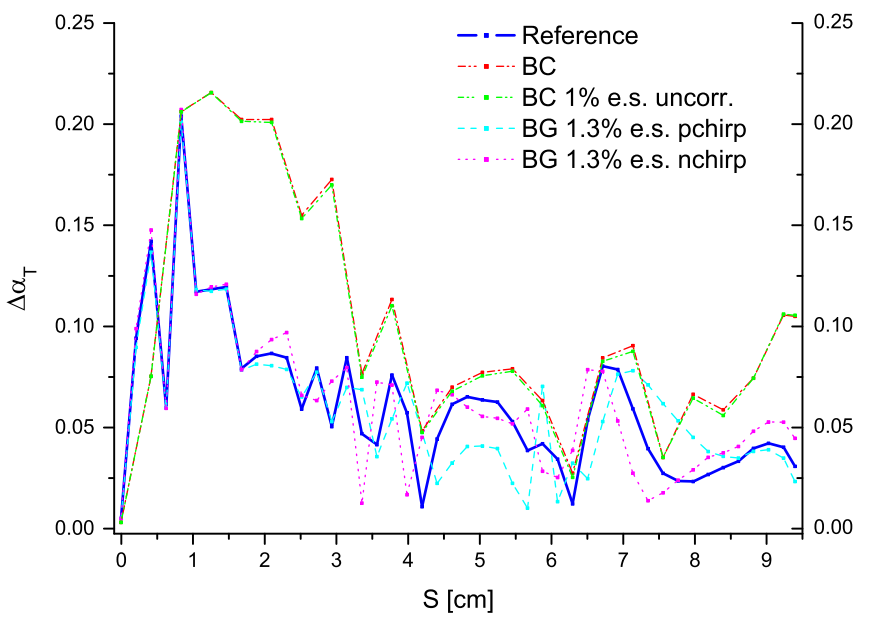

Fig. 7. Spread in $\alpha_{\mathrm{T}}$ for the distributions considered in Fig. 5. See main text for details.

tails in Gaussians) and further shows how different is the dynamics from that described in [25].

It also explains the better performance, in terms of emittance, of the BC distribution: the current profile rises in a much sharper way than a BG distribution; this makes the beam driven plasma wave front hedge closer to the bunch head, while transversally its extent is not much different than in the BG situation, since the parameter $\tilde{Q}=Q_{\text {bunch }} k_{p}^{3}$ [26], valid for bunches smaller than $\lambda_{p}$ in any given direction, is the same in both settings and much smaller than one. Due to the bunch solid hedges, the overall number of particles found in the weak focusing and transition region is larger than in the case of the BG distribution; this explains why the first emittance peak in Fig. 5 is higher for the BC distribution, compared to the RS. However, the BC lacks the tails of the BG, so the drifting particles are more effectively re-captured in the laser driven wake along the acceleration section and remain, generally speaking, closer to the axis. The emittance compensation is then faster and more effective in the extraction ramp.

Following the same reasoning it is possible to understand that a residual ballistic focusing can reduce the amount of charge drifting in neutral plasma at injection, as already shown in [13] in absence of beam loading; the overall converging transverse momentum helps in keeping the particles within the self generated wake and reduces the drift extent of those outside it. This is supported by the fact that the emittance peak is lower and a little bit more downstream than in the $\alpha_{\mathrm{T}}=0$ situation (Fig. 4). Given a better insertion, the subsequent transport is consequentially improved. A larger negative value of $\alpha_{\mathrm{T}}$, however, does not improves emittance, since the ripples in the acceleration stage are larger and with increasing amplitude.

\section{Conclusions}

In this paper we extended the study of the matching strategy proposed in [14], assessing its robustness against jitters in matching conditions: small variations in the beam parameters always produce small variations in the final beam spot-size. Emittance turns out to be more sensitive to some jitters than others: for example reducing the beam size by $10 \%$ is much more detrimental than increasing it by the same quantity. We also checked how the transport results are modified by the beam charge configuration, showing that bi-Gaussian and double flat-top (beer can) distributions yield comparable results. We conclude that the matching strategy is reasonably robust against reasonable jitters that can be present in a realistic experimental setting and can be fruitfully exploited to accelerate high brightness electron bunches.

Finally, we analyzed the emittance evolution and demonstrated how its variations in the insertion and extraction ramps can be explained by the interplay between transverse 'slices' and beam loading effects.

All results depends on the specific setting under examination and are expected to be valid in a neighborhood of the starting energy and emittance.

\section{Acknowledgments}

This work has been partially supported by the EU Commission in the Seventh Framework Program, Grant Agreement 312453 EUCARD-2 and by the Italian Ministry of Research in the framework of FIRB - Fondo per gli Investimenti della Ricerca di Base, Project no. RBFR12NK5K.

\section{References}

[1] T. Tajima, J.M. Dawson, Physical Review Letters 43 (1979) 267.

[2] M. Litos, et al., Nature 515 (2014) 92;

(a) W. Leemans, et al., Nature Physics 2 (2006) 696;

(b) I. Blumenfeld, et al., Nature 445 (2007) 741;

(c) S. Kneip, et al., Physical Review Letters 103 (2009) 035002

(d) D.H. Froula, et al., Physical Review Letters 103 (2009) 215006;

(e) C.E. Clayton, et al., Physical Review Letters 105 (2010) 105003;

(f) X. Wang, et al., Nature Communications 4 (2013) 1988.

[3] M. Veysman, et al., Journal of the Optical Society of America B 27 (2010) 1400; (a) M. Veysman, et al., Physical Review E 86 (2012) 066411;

(b) N.E. Andreev, et al., Nuclear Instruments and Methods in Physics Research A 740 (2014) 273.

[4] E. Esarey, C. Schroeder, W. Leemans, Reviews of Modern Physics 81 (2009) 1229;

(a) M. Ferrario, in: proceedings of the International school of physics Enrico Fermi in Laser-plasma acceleration, Course 179. Varenna on Lake Como, Villa Monastero, 20-25 June 2011.

[5] P. Antici, et al., Journal of Applied Physics 112 (2012) 044902.

[6] K. Floettmann, Physical Review Special Topics Accelerators and Beams 6 (2003) 034202.

[7] M. Migliorati, et al., Physical Review Special Topics Accelerators and Beams 16 (2013) 011302

[8] J. van Tilborg, et al., Physical Review Letters 115 (2015) 184802.

[9] R. Lehe, et al., Physical Review Special Topics Accelerators and Beams 17 (2014) 121301.

[10] C.M.S. Sears, et al., Physical Review Special Topics Accelerators and Beams 13 (2010) 092803.

[11] R. Weingartner, et al., Physical Review Special Topics Accelerators and Beams 15 (2012) 111302.

[12] X.L. Xu, et al, arXiv:1411.4386v2 [physics.acc-ph], 2015.

[13] I. Dornmair, et al., Physical Review Special Topics Accelerators and Beams 18 (2015) 041302.

[14] P. Tomassini, A.R. Rossi, Plasma Physics and Controlled Fusion 58 (2016) 034001.

[15] T. Mehrling, et al., Physical Review Special Topics Accelerators and Beams 15 (2012) 111303

[16] K. Floettmann, Physical Review Special Topics Accelerators and Beams 17 (2014) 054402.

[17] M. Reiser, Theory and Design of Charged Particle Beams, second ed., WILEYVCH Verlag GmbH \& Co. KGaA, Weinheim, 1994.

[18] A.R. Rossi, et al., Nuclear Instruments and Methods in Physics Research A 740 (2014) 60.

[19] W. Leemans, E. Esarey, Physics Today 62 (2009) 44.

[20] J.K. Lim, Physical Review Special Topics Accelerators and Beams 8 (2005) 072401.

[21] B. Cros, et al., Physical Review E 65 (2002) 026405.

[22] J. Krall, et al., Physics of Plasmas 1 (1994) 1738.

[23] C. Benedetti, et al., IEEE Transactions on Plasma Science 36 (2008) 1790.

[24] K. Floettmann, ASTRA, 〈http://desy.de/mpyflo/Astra_dokumentation/.

[25] B.E. Carlsten, Nuclear Instruments and Methods in Physics Research A 285 (1989) 311;

(a) L. Serafini, J.B. Rosenzweig, Physical Review E 55 (1997) 7565; (b) M. Ferrario, et al., Physical Review Letters 99 (2007) 234801.

[26] J.B. Rosenzweig, et al., Physical Review Special Topics Accelerators and Beams 7 (2004) 061302. 